\title{
Morbi-Mortalidad Materna y Perinatal en Pacientes con Pre-Eclampsia y Eclampsia
}

\author{
Dr. Alfonso Jubiz $H^{*}$ \\ Dr. William Mejía V*
}

Dra. Betty Medina S. **

\section{INTRODUCCION}

La hipertensión que se desarrolla durante el embarazo es una de las principales causas de muerte en Obstetricia. En Inglaterra, la pre-eclampsia y la eclampsia ocupan el tercer lugar después del aborto y del embolismo pulmonar (5). Entre nosotros es la tercera causa (6).

En los países más desarrollados la incidencia de la pre-eclampsia y la eclampsia se ha logrado disminuir gracias a la selección meticulosa de los factores de riesgo y además, al manejo prenatal adecuado. Sin embargo, existe un grupo de embarazadas que no pueden librarse de esta patología. Con una consulta prenatal bien orientada, del 2 al $3 \%$ de las muertes perinatales se deben a la hipertensión que se desarrolla durante el embarazo (9). Los efectos que la elevación de la tensión arterial produce en los diferentes sistemas de la madre, afectan la salud del feto.

Uno de los principales factores que inciden en la prevención de la morbimorta-

* Profesores titulares. Facultad de Medicina de la Universidad de Antioquia.

* * Médica Ginecobstetra. lidad es la dificultad que se presenta para reconocer en una etapa temprana de la gestación los cambios mínimos de la tensión arterial. Por lo anterior, se ha recomendado la utilización de la prueba de. Gant (4) para seleccionar el grupo de pacientes predispuestas.

El manejo terapéutico de esta patología, es la consecuencia lógica de la causa que lo desencadena, la cual, hasta el presente, continúa en la oscuridad.

El régimen terapéutico inicial de este síndrome, se enfocó siempre hacia el control $y$ tratamiento de la madre. Hoy, se persiguen tres objetivos que son:

1. Prevenir el desarrollo de las convulsiones.

2. Obtener un recién nacido en buenas condiciones, $y$

3. Evitar que la hipertensión deje secuelas en la madre.

Por lo tanto, se ha hecho énfasis en el estudio de la función placentaria y en el desarrollo del feto, razón por la cual la ultrasonografía, los estudios endocrinos, enzimáticos y bioeléctricos, se incrementan cada día y son indispensables en la va- 
loración fetal de toda paciente con preeclampsia y eclampsia.

\section{Objetivos}

Los objetivos específicos fueron:

1. Determinar el estado final de las madres que padecieron pre-eclampsia o eclampsia y que fueron atendidas en el Departamento de Obstetricia y Ginecología durante los años 1974 a 1978.

2. Determinar el estado inicial y final de los recién nacidos del grupo de madres en estudio.

3. Determinar el porcentaje de atención prenatal.

4. Discriminar la forma de terminación del embarazo.

\section{Material y métodos}

Se revisaron todas las historias clínicas de las pacientes con el diagnóstico de preeclampsia y eclampsia que fueron atendidas en el Departamento de Obstetricia y Ginecología entre el primero de enero de 1974 y el 31 de diciembre de 1978.

Se aceptó como definición de preeclampsia la siguiente:

Síndrome que aparece durante la gestación, después de la semana 24 , caracterizado por: hipertensión de 140/90 para la moderada y de $160 / 100$ para la grave, además, edema y albuminuria.

Eclampsia: El mismo cuadro anterior acompañado de convulsiones o coma.
No se incluyó en este estudio a aquellas pacientes con historia de hipertensión, enfermedad renal o vascular.

Se utilizó un sistema de tabulación manual con tarjetas Mac-Bee y las variables registradas fueron las siguientes:

1. Año de hospitalización

2. Edad de la madre

3. Número de embarazos

4. Número de partos

5. Número de hijos vivos

6. Edad gestacional al ingreso y en el mo. mento de la terminación del embarazo.

7. Estado del feto al ingreso

8. Diagnóstico.

9. Forma de terminación del embarazo

10. Estado del recién nacido al minuto, a los cinco minutos $y$ al salir del Hospital.

\section{Complicaciones maternas}

12. Estado de la madre al salir del Hospital.

Se revisaron 172 historias y después de excluir las que no llenaron los requisitos quedaron 129 que constituyen el universo del estudio.

Durante el lapso mencionado se registraron 30.896 egresos. El número de pacientes atendidas por año es el siguiente: 


$\begin{array}{lll}1974 & 30 & \begin{array}{l}\text { das provienen de otros municipios, razón } \\ \text { por la cual se desconocen algunos datos } \\ \text { de la Historia Clínica. }\end{array}\end{array}$

$1976 \quad 34$

$1977 \quad 25$

$1978 \quad 21$

El Hospital Universitario San Vicente de Paúl es un centro asistencial de referencia, y la mayoría de las pacientes atendi-

\section{Resultados}

El análisis de las historias permitió obtener los datos que aparecen a continuación:

La distribución porcentual de la clasificación aparece en el Cuadro No. 1

CUADRO No. 1

\section{Clasificación}

\begin{tabular}{ccc}
\hline Diagnóstico & No. & $\%$ \\
\hline Pre-eclampsia moderada & 46 & 35.65 \\
Pre - eclampsia grave & 50 & 38.75 \\
Eclampsia & 33 & 25.60 \\
\hline Total & 129 & 100.00 \\
\hline
\end{tabular}

De las pacientes con eclampsia, 9 ocurrieron en el post - parto. El porcentaje de las eclampsias es muy alto a pesar de ser la mitad de la pre - eclampsia.

El grupo más numeroso fue el de 20 a 24 años con 44 casos $(34.1 \%)$. Sin embargo, la edad no muestra ninguna relación con los diferentes grupos de toxemia.
Si analizamos los diagnósticos antes de las 35 semanas podemos observar que en la pre-eclampsia moderada, el $17.7 \%$ tenía esa edad gestacional, en la pre-eclampsia grave el $35.7 \%$ y en la eclampsia el $54.5 \%$. Lo anterior nos manifiesta que entre más precoz es la aparición del síndrome, más grave es su evolución. Por lo tanto la consulta prenatal se debe iniciar en una etapa precoz del embarazo. 
CUADRO No. 2

Edad gestacional al ingreso según el diagnóstico

\begin{tabular}{cccccc}
\hline E dad gestacional & Moderada & Grave & Eclampsia & Total \\
\hline $24-27$ & - & 4 & 4 & 8 \\
$28-$ & 31 & - & 1 & 6 & 7 \\
$32-35$ & -31 & 10 & 2 & 20 \\
$36 \quad-\quad 39$ & 21 & 20 & 7 & 48 \\
$40 \quad$ y más & 16 & 7 & 3 & 26 \\
Desconocida & 1 & 8 & 11 & 20 \\
\hline Total & 46 & 50 & 33 & 129 \\
\hline
\end{tabular}

CUADRO No. 3

Consulta prenatal según el diagnóstico

\begin{tabular}{lcccccccc}
\hline \multicolumn{1}{c}{ Diagnóstico } & Si & $\%$ & No & $\%$ & $\begin{array}{c}\text { Descono- } \\
\text { cido }\end{array}$ & $\%$ & Total & $\%$ \\
\hline P. Moderada & 36 & 78.3 & 8 & 17.4 & 2 & 4.3 & 46 & 100.0 \\
P. Grave & 26 & 52.0 & 19 & 38.0 & 5 & 10.0 & 50 & 100.0 \\
Eclampsia & 8 & 24.1 & 20 & 60.6 & 5 & 15.2 & 33 & 100.0 \\
\hline Total & 70 & 54.3 & 47 & 36.4 & 12 & 9.3 & 129 & 100.0 \\
\hline
\end{tabular}

El $36.4 \%$ no asistieron a la consulta prenatal, y si sumamos el $9.3 \%$ en las cuales se desconoce el dato se podría suponer que el $45.7 \%$ no tuvieron el privilegio de recibir una atención preparto. Además de las pacientes que sí la recibieron, el $90 \%$ sólo asistió a una o dos consultas, las cuales se efectuaron generalmente, para remitirlas al Hospital. Unicamente dos pacientes tuvieron cinco y seis consultas respectivamente. A la escasa atención prenatal se le suma la deficiente calidad en estas dos que tuvieron un número aceptable de contioles. 
CUADRO No. 4

Estado fetal al ingreso según el diagnóstico

\begin{tabular}{lccccccc}
\hline Diagnóstico & Vivo & $\%$ & Muerto & $\%$ & $\begin{array}{c}\text { Descono- } \\
\text { cido }\end{array}$ & $\%$ & Total \\
\hline P. Moderada & 45 & 39.8 & 1 & 9.1 & - & - & 46 \\
P. Grave & 48 & 42.5 & 3 & 27.3 & 3 & 33.3 & $54^{*}$ \\
Eclampsia & 20 & 17.7 & 7 & 63.6 & 6 & 66.7 & 33 \\
\hline Total & 113 & 100.0 & 11 & 100.0 & 9 & 100.0 & $133^{*}$ \\
$\%$ & 85.0 & - & 8.3 & - & 6.7 & - & \\
\hline
\end{tabular}

* 4 Gemelos

En la eclampsia se aprecia una tasa de fetos muertos al ingreso de 350 por mil y de 97 por mil para el total de casos. Las anteriores cifras nos indican la gravedad del proceso desde el punto de vista fetal.

CUADRO No. 5

Terminación del embarazo según el diagnóstico

\begin{tabular}{lccccccccc}
\hline Diagnóstico & $\begin{array}{c}\text { Parto } \\
\text { espontáneo }\end{array}$ & $\%$ & Fórceps & $\%$ & Espátulas & $\%$ & Cesárea & $\%$ & Total \\
\hline P. Moderada & 32 & 48.5 & 2 & 12.5 & - & - & 12 & 24.0 & 46 \\
P. Grave & 19 & 28,8 & 10 & 62.5 & 1 & - & 24 & 48.0 & $54^{*}$ \\
Eclampsia & 15 & 27.7 & 4 & 25.0 & - & - & 14 & 28.0 & 33 \\
\hline Total & 66 & 100.0 & 16 & 100.0 & 1 & - & 50 & 100.0 & $133^{*}$ \\
$\%$ & 49.6 & & 12.0 & & 0.8 & & 37.6 & & \\
\hline
\end{tabular}

\section{* 4 Gemelos}

Llama la atención el porcentaje tan al to de partos espontáneos $(49.6 \%$ ) y el escaso número de fórceps y espátulas $(12.8 \%)$. El porcentaje de cesáreas es bajo si tenemos en cuenta la tendencia actual de practicar más extracciones fetales por vía abdominal en los casos de pre-eclampsia grave y eclampsia. 
CUADRO No. 6

Indice de Apgar según el diagnóstico. Un minuto

\begin{tabular}{lcccccc}
\hline Diagnóstico & $\begin{array}{r}\text { Nacido } \\
\text { muerto }\end{array}$ & $0-3$ & $4-6$ & $7-10$ & $\begin{array}{c}\text { Descono- } \\
\text { cido }\end{array}$ & Total \\
\hline P. Moderada & 1 & 5 & 1 & 36 & 3 & 46 \\
P. Grave & 3 & 5 & 7 & 29 & 10 & $54^{*}$ \\
Eclampsia & 7 & 3 & 6 & 7 & 10 & 33 \\
Total & 11 & 13 & 14 & 72 & 23 & $133^{*}$ \\
$\%$ & 8.3 & 9.8 & 10.5 & 54.1 & 17.3 & 100.0 \\
\hline
\end{tabular}

* 4 Gemelos

En el $34.5 \%$ de los casos conocidos, el Apgar al primer minuto fue menor de 7, este dato es muy elevado al hacer el análisis global. Sin embargo, en la eclampsia esta cifra es mucho más alta $(67.6 \%)$.

CUADRO No. 7

Indice de Apgar según el diagnóstico. Cinco minutos

\begin{tabular}{lcccccc} 
Diagnóstico & $\begin{array}{r}\text { Nacido } \\
\text { muerto }\end{array}$ & $0-3$ & $4-6$ & $7-10$ & $\begin{array}{c}\text { Descono- } \\
\text { cido }\end{array}$ & Total \\
\hline P. Moderada & 1 & 3 & 3 & 36 & 3 & 46 \\
P. Grave & 3 & 4 & 4 & 39 & 4 & $54^{*}$ \\
Eclampsia & 7 & 2 & 4 & 9 & 11 & 33 \\
Total & 11 & 9 & 11 & 84 & 18 & $133^{*}$ \\
$\%$ & 8.3 & 6.8 & 8.3 & 63.1 & 15.5 & 100.0 \\
\hline
\end{tabular}

* 4 Gemelos

En el Cuadro No. 7 sólo podemos destacar que en la preclampsia grave, la recuperación del recién nacido a los cinco minutos es mayor que en los otros grupos. 
CUADRO No. 8

Peso en gramos del recién nacido según el diagnóstico

\begin{tabular}{lccccccc}
\hline Diagnóstico & $\begin{array}{c}1.000 \\
a\end{array}$ & $\begin{array}{c}1.500 \\
a\end{array}$ & $\begin{array}{c}2.000 \\
a\end{array}$ & 2.500 & Desconocido & Total \\
& 1.499 & 1.999 & 2.499 & $y$ más & & \\
\hline P. Moderada & - & 5 & 6 & 35 & - & 46 \\
P. Grave & 2 & 7 & 10 & 33 & 2 & $54^{*}$ \\
Eclampsia & 7 & 3 & 5 & 8 & 10 & 33 \\
\hline Total & 9 & 15 & 21 & 76 & 12 & $133^{*}$ \\
$\%$ & 6.8 & 11.3 & 15.8 & 57.1 & 9.0 & 100.0 \\
\hline
\end{tabular}

* 4 Gemelos

En los casos de eclampsia en los cuales aparece el dato del peso, el $34.7 \%$ sobrepasaron los 2.500 gramos. Este porcentaje contrasta con el $60 \%$ de los otros grupos.

CUADRO No. 9

Estado del recién nacido a la salida según el diagnóstico

\begin{tabular}{lcccc}
\hline Diagnóstico & Vivo & Muerto & Desconocido & Total \\
\hline P. Moderada & 43 & 1 & 2 & 46 \\
P. Grave & 47 & 4 & 3 & $54^{*}$ \\
Eclampsia & 20 & 7 & 6 & 33 \\
\hline Total & 110 & 12 & 11 & $133^{*}$ \\
$\%$ & 82.7 & 9.0 & 8.3 & 100.0 \\
\hline
\end{tabular}

* 4 Gemelos

El $82.7 \%$ de los recién nacidos salieron vivos del Hospital. Durante el tratamiento murió uno y se desconoce la evolución de dos casos. 


\section{Complicaciones maternas}

Se presentaron cuatro complicaciones maternas y tres de ellas corresponden al grupo de eclampsia. Murieron tres pacientes, dos por accidente cerebro-vascular $y$ en la otra no se pudo esclarecer el diagnóstico. La otra complicación encontrada fue una insuficiencia renal aguda que mejoró con diálisis peritoneal. La mortalidad en la eclampsia fue de 23.3 por mil y la global de 31 por mil.

\section{Discusión}

La hipertensión arterial que complica el embarazo continúa ocupando un sitio importante y significa un riesgo tanto para la madre como para el feto. A pesar del subregistro tan común en nuestras estadísticas la tasa global durante los cinco años fue de 4.2 por mil egresos hospitalarios.

Sullivan (7) anota que la incidencia en Estados Unidos en varios grupos de primíparas fluctuó entre 7 y $30 \%$. Hibbard $y$ Rosen (5) informaron que entre ellos, la incidencia ha disminuido en relación con los partos de $2.3 \%$ en el período 1965 1969 a $0.7 \%$ entre el 70 y el 74 . Estas cifras están relacionadas con los criterios de selección y metodología de cada estudio.

La gran mayoría de las pacientes analizadas está constituida por primigestantes $(61.2 \%)$, dato completamente demostrado $y$ que nos obliga a una mayor vigilancia prenatal en este grupo vulnerable. Además, el $75 \%$ tuvieron una edad inferior a 24 años y una tenía 14.
Nuestro centro hospitatario es un lugar de referencia de pacientes de otros municipios diferentes a Medellín y aún, de los departamentos vecinos. Además, al igual que muchos hospitales colombianos tiene limitaciones locativas y escasez de recurso humano y económico. El hallazgo de 11 fetos muertos al ingreso (97 por mil) nos da una idea de la gravedad del proceso en relación con el producto. Es difícil evaluar las condiciones en las cuales se hizo el manejo de estas pacientes, los criterios fueron diferentes y las complicaciones bien podrían deberse ai enfoque terapéutico inicial. Durante la hospitalización murió un recién nacido y se desconoce la evolución de otro.

La mortalidad perinatal no corregida de 82.7 por mil nacidos vivos para el grupo estudiado, nos debe preocupar y a la vez motivar para mejorar los sistemas de selección y control de las pacientes de riesgo, para lograr en un futuro la disminución de esta mortalidad. Si hacemos la corrección con base en la exclusión de los que llegaron muertos, la tasa de mortalidad perinatal es de 8 por mil.

Tejani y col (8) informan una mortalidad perinatal en pre-eclampsia y eclampsia de 75 por mil entre 1970 y 1972, con una disminución a 28 por mil no corregida y de 12 corregida, entre 1973 y 1975. Anderson (1) con un tratamiento conservador encontró una mortalidad corregida de $7 \%$

La mortalidad materna de 23.3 por mil para la eclampsia y de $\mathbf{3 1}$ para todo el grupo nos indica que esta patología desempeña un papel primordial en las muertes maternas. Ya mencionamos cómo la mayoría de estas pacientes son referidas de 
otros hospitales del Departamento. Anderson ya citado, encontró una muerte materna sobre 246 pre-eclampsias y 13 eclampsias. En Medellín se registraron 2 muertes maternas por toxemia en el año 1977 y la mortalidad fue de 1.4 por mil.

En el mismo año, en Antioquia se consignan 14 muertes por la misma causa y corresponde a una mortalidad materna de 3.2 por mil. (2).

Es necesario aclarar que las tasas anotadas se refieren únicamente a la hipertensión que complica la gestación.
Indiscutiblemente, la base primordial para disminuir la incidencia y complicaciones de esta patología, es la consulta prenatal bien orientada. Prácticamente dos pacientes tuvieron un número adecuado de controles. La atención prenatal debe ser precoz, de buena calidad, con la frecuencia necesaria $y$ también selectiva para aquellas gestantes predispuestas.

El análisis de este grupo de pacientes nos debe servir para corregir todos los errores que fácilmente se cometen alrededor del proceso reproductivo.

\section{MOTHER AND PERINATAL MORBI-MORTALITY IN PATIENTS WITH PRE-ECLAMPSIA AND ECLAMPSIA}

\section{Summary}

An analysis was made of all of the 129 records of patients with pre-eclampsia and eclampsia that were hospitalized in the Obstetrics and Ginecology Department of Hospital Universitario San Vicente de Paul in Medellin (Colombia) from January 1974 through December 1978.

$46(35.65 \%)$ patients had moderate pre-eclampsia, $50(38.75 \%)$ were seriously ill and $33(25.60 \%)$ showed eclampsia. $7 \%$ of these appeared in the puerperium.

Three patients died, two of which had a cerebrovascular accident.

The total number of newborns was 133, 11 entered dead, one died during hospitalization and the evolution of two remain unknown. Uncorrected perinatal mortality was of 82.7 per thousand and of
8 per thousand for corrected. $49.6 \%$ had a normal and spontaneous delivery and spontaneous delivery and $37.6 \%$ finished pregnancy through caesarean operation. The percentage of prenatal control was of $36.48 \%$.

\section{Resumen}

Se analizaron 129 historias correspondientes a la totalidad de las pacientes con pre-eclampsia y eclampsia que estuvieron hospitalizadas en el Departamento de Obstetricia y Ginecología del Hospital Universitario San Vicente de Paúl, de Medellín (Colombia) desde enero de 1974 a diciembre de 1978.

El total de pacientes con pre-eclampsia moderada fue de $46(35.65 \%)$, graves 50 $(38.75 \%)$ y eclampsia $33(25.60 \%)$. De éstas, el $7 \%$ se presentaron en el puerperio. 
Murieron 3 pacientes, de las cuales dos presentaron un accidente cerebro-vascular.

El número de recién nacidos fue de 133, 11 ingresaron muertos, uno murió durante la hospitalización y se desconoce la evolución de dos. La mortalidad perinatal fue de 82.7 por mil no corregiday de 8 por mil corregida. El $49.6 \%$ tuvieron un parto normal y espontáneo y $37.6 \%$ terminaron el embarazo por cesárea. El porcentaje de control prenatal fue de $36.4 \%$

\section{Bibliografía}

1. ANDERSON, W. A. and HARBERT, G. $M$. Jr. Convervative management of preeclamptic and eclamptic patients: $A$ reevaluation. Am. J. Obstet. Gynecol. 1 129 (3): 260 - 266, 1977.

2. Anuario Estadístico de Antioquia. DANE 1977.
3. FERRIS, T. F. Toxemia and hypertension In Medical complications during pregnancy. Edited by Burrow and Ferris. Philadelphia. Saunders. 1975 pp. 53-104.

4. GANT, N. F. et. al. A clinicas text useful for predicting the development of acute hypertension in pregnancy. Am. J. Obstet. Gynecol. 120: 1, 1974.

5. HIBBARD, B. M. and ROSEN, M. The management of severe pre-eclampsia and eclampsia. Br. J. Angesth. 49 (3): 3-9, 1977.

6. JUBIZ, H. A. y SANCHEZ, M. C. Muertes maternas en el Hospital Universitario San Vicente de Paúl. Rev. Colomb. Obstet. Ginecol. 29 (4): 178-182, 1978.

7. SULLIVAN, J. M. Hypertension inpregnancy. Clin. Perinatol. 1 (2); 369-384, 1974.

8. TEJANI, S. N. Modern management of hypertensión disorders of pregnancy. Clin. Obstet. Gynecol. 51 (6): 648-653, Jun. 1978.

9. WELT, S. W. and CRENSHAN, M. C. Concurrent hypertension and pregmancy Clin. Obstet. Gynecol. 21 (3): 619-648, 1978. 